\title{
Other B decays at Belle
}

\section{Min-Zu Wang ${ }^{* i}$}

National Taiwan University, Taiwan

E-mail: mwang@phys.ntu.edu.tw

Recent results of Cabbibo-suppressed and color-suppressed $B$ decays from Belle are reported. We observe clear signal for a Cabibbo suppressed decay mode $B^{-} \rightarrow \bar{p} \Lambda D^{0}$, but no significant signal is found in a similar decay mode, $B^{-} \rightarrow \bar{p} \Lambda D^{* 0}$. We update the measurement of a color suppressed decay mode $B^{0} \rightarrow J / \psi \eta$, but similarly no significant signal is found in a similar decay mode, $B^{0} \rightarrow J / \psi \eta^{\prime}$. This study is based on a large data sample collected by the Belle detector at the KEKB asymmetric energy $e^{+} e^{-}(3.5$ on $8 \mathrm{GeV})$ collider.

The 2011 Europhysics Conference on High Energy Physics-HEP 2011,

July 21-27, 2011

Grenoble, Rhône-Alpes France

\footnotetext{
* Speaker.

$\dagger$ on behalf of the Belle Collaboration
} 


\section{Introduction}

The dominant process for $B$ meson decays is via the $b \rightarrow c$ tree diagram at quark level. However, due to the constraint on the quark contents of final state particles, some $b \rightarrow c$ decays are Cabbibo-suppressed or color-suppressed. The decay branching fractions of these suppressed modes are typically around $\sim 10^{-5}$, and some interesting phenomena could be expected due to the interference effect between different amplitudes of comparable magnitude. Since these decay amplitudes should be suppressed, if unexpected large decay branching fractions are observed, it is a strong evidence indicating the existence of long distance effect via the final state re-scattering.

In this presentation, we will report recent results of Cabbibo-suppressed and color-suppressed $B$ decays from Belle. These include the measurements of the $B$ decay branching fractions for $B^{-} \rightarrow \bar{p} \Lambda D^{(*) 0}[1,2]$ and $B^{0} \rightarrow J / \psi \eta^{\left({ }^{\prime}\right)}$. Studying the former decay process could be useful to understand the polar angle anomaly found at the baryon-antibaryon rest frame in $B^{+} \rightarrow p \bar{p} K^{+}$[3] and $B^{0} \rightarrow p \bar{\Lambda} \pi^{-}$[4]. The above three-body decays are believed to proceed dominantly through the $b \rightarrow s g$ strong penguin process and the $b \rightarrow u$ tree process, where $g$ stands for the hard gluon. This strong penguin process is sensitive to new physics due to possible contributions from heavy unknown particles in the penguin loop. It is suggested that one can look into the $b \rightarrow c$ dominant decays first [5] since the penguin contribution is negligible. In this way, one can estimate the $b \rightarrow u$ contribution and dis-entangle the underlying dynamics. Based on a generalized factorization approach, the predicted branching fraction is about $10^{-5}$ for $B^{-} \rightarrow \bar{p} \Lambda D^{0}$ and $B^{-} \rightarrow \bar{p} \Lambda D^{* 0}$. They are already in the reach of the accumulated data at Belle [6].

The $B^{0} \rightarrow J / \psi \eta$ decay has been observed [7] before and the measured branching fraction is about $10^{-5}$. Assuming the $\eta-\eta^{\prime}$ mixing angle in the octet-singlet basis is $(40.4 \pm 0.6)^{\circ}$ [8], the expected branching fraction for $B^{0} \rightarrow J / \psi \eta^{\prime}$ will be about $70 \%$ of that of $B^{0} \rightarrow J / \psi \eta$. It is again in the reach of the accumulated data at Belle.

\section{Results of $B^{-} \rightarrow \bar{p} \Lambda D^{0}$ and $B^{-} \rightarrow \bar{p} \Lambda D^{* 0}$}

The data sample used in this study corresponds to an integrated luminosity of $605 \mathrm{fb}^{-1}$ and contains $657 \times 10^{6} B \bar{B}$ pairs collected with the Belle detector on the $\Upsilon(4 \mathrm{~S})$ resonance at the KEKB asymmetric-energy $e^{+} e^{-}(3.5 \mathrm{GeV}$ and $8 \mathrm{GeV})$ collider [9]. The selection criteria for the final state charged particles of $B^{-} \rightarrow \bar{p} \Lambda D^{0}$ are based on information obtained from the tracking system and the particle identification system. We reconstruct $\Lambda$ from its sub-decay mode, $\Lambda \rightarrow p \pi^{-}$, and reconstruct $D^{0}$ mesons from $D^{0} \rightarrow K^{-} \pi^{+}$and $D^{0} \rightarrow K^{-} \pi^{+} \pi^{0}$. The $\pi^{0}$ candidates are formed by two isolated photon clusters measured by the electromagnetic calorimeter.

We use two kinematic variables in the center of mass frame to identify the reconstructed $B$ meson candidates: the beam energy constrained mass $M_{\mathrm{bc}}=\sqrt{E_{\text {beam }}^{2}-p_{B}^{2}}$, and the energy difference $\Delta E=E_{B}-E_{\text {beam }}$, where $E_{\text {beam }}$ is the beam energy, and $p_{B}$ and $E_{B}$ are the momentum and energy, respectively, of the reconstructed $B$ meson. We perform an unbinned extended likelihood fit using the information from $M_{\mathrm{bc}}$ and $\Delta E$ of each candidate event to extract the signal yields. Fig. 1 shows the fit result for $B^{-} \rightarrow \bar{p} \Lambda D^{0}$. 
The branching fractions are calculated by using the formula

$$
\mathscr{B}=\frac{N_{\text {signal }}}{\varepsilon \times f \times N_{B \bar{B}}},
$$

where $N_{\text {signal }}, N_{B \bar{B}}, \varepsilon$ and $f$ are the number of signal yield, the number of $B \bar{B}$ pairs, the reconstruction efficiency, and the relevant sub-decay branching fractions like $\mathscr{B}\left(\Lambda \rightarrow p \pi^{-}\right)=63.9 \pm 0.5 \%$, $\mathscr{B}\left(D^{0} \rightarrow K^{-} \pi^{+}\right)=3.89 \pm 0.05 \%$, and $\mathscr{B}\left(D^{0} \rightarrow K^{-} \pi^{+} \pi^{0}\right)=13.9 \pm 0.5 \%$. The branching fraction obtained is $\left(1.43_{-0.25}^{+0.28} \pm 0.18\right) \times 10^{-5}$. We also observe low mass enhancements near threshold which is a common feature for charmless three-body baryonic $B$ decays. The measured branching fraction agrees well with the theoretical prediction [5]. The measured branching fraction can be used to tune the parameters in the event generator, e.g. Pythia, for the fragmentation process involving $b$ quarks.

We study $B^{-} \rightarrow \bar{p} \Lambda D^{* 0}$ with $D^{* 0} \rightarrow D^{0} \pi^{0}$ and $D^{0} \rightarrow K^{-} \pi^{+}$. Fake (mostly soft) $\pi^{0}$ 's combined with true $\bar{p} \Lambda D^{0}$ signals from $B^{-} \rightarrow \bar{p} \Lambda D^{* 0}$ or $\bar{B}^{0} \rightarrow \bar{p} \Lambda D^{*+}$ can cause signal-like peaking feature in $M_{\mathrm{bc}}$ and $\Delta E$. One needs to use the information from the mass difference between $D^{* 0}$ and $D^{0}$, $\Delta M$, in order to separate signal and background. Since the signal yield is not significant after the $\Delta M$ background subtraction, we determine the upper limit $\mathscr{B}\left(B^{-} \rightarrow \bar{p} \Lambda D^{* 0}\right)<4.8 \times 10^{-5}$ at the $90 \%$ confidence level by the Feldman-Cousin method [10]. With the current statistics, this upper limit agrees with the theoretical prediction which is about $3 \times 10^{-5}$.

(a)
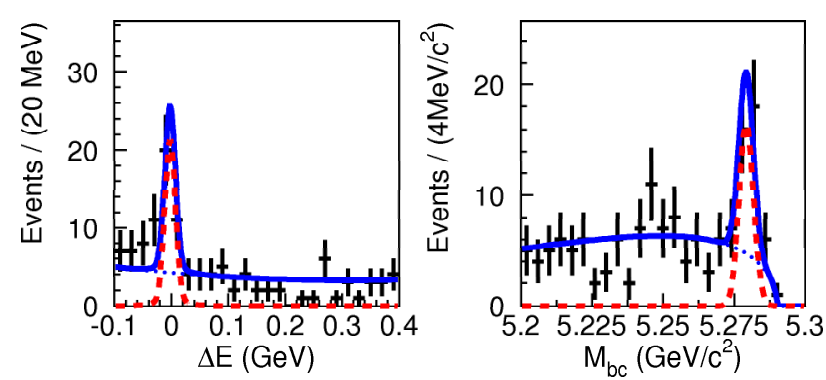

(b)
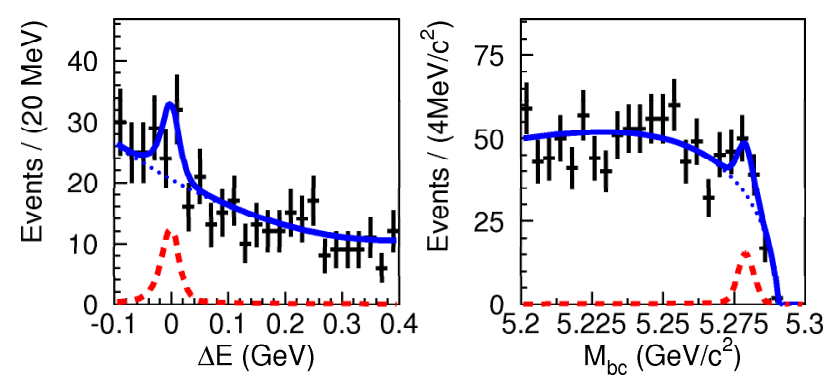

Figure 1: Fit results for (a) $B^{-} \rightarrow \bar{p} \Lambda D^{0}, D^{0} \rightarrow K^{-} \pi^{+} \pi^{0}$ and (b) $B^{-} \rightarrow \bar{p} \Lambda D^{0}, D^{0} \rightarrow K^{-} \pi^{+} \pi^{0}$. The solid curve shows the fit; the peaking curve represents signal, and the dotted line indicates background.

\section{Results of $B^{0} \rightarrow J / \psi \eta$ and $B^{0} \rightarrow J / \psi \eta^{\prime}$}

The data sample used in this $B^{0} \rightarrow J / \psi \eta^{\left({ }^{\prime}\right)}$ study is $711 \mathrm{fb}^{-1}$ which contains $772 \times 10^{6} B \bar{B}$ 
pairs. This corresponds to the full data set collected by Belle at the $\Upsilon(4 \mathrm{~S})$ resonance. We select the $J / \psi$ particle using its leptonic decay modes since they have very good background rejection power. We reconstruct $\eta$ with $\eta \rightarrow \gamma \gamma$ and $\eta \rightarrow \pi^{+} \pi^{-} \pi^{0}$, and reconstruct $\eta^{\prime}$ with $\eta^{\prime} \rightarrow \rho^{0} \gamma$ and $\eta^{\prime} \rightarrow \eta \pi^{+} \pi^{-}$. Due to the consideration of background, we only use the $\eta \rightarrow \gamma \gamma$ mode in the reconstruction of $\eta^{\prime} \rightarrow \eta \pi^{+} \pi^{-}$.

We change the fitting procedure by requiring the reconstructed $B$ candidates to have $M_{\mathrm{bc}}>5.27$ $\mathrm{GeV} / c^{2}$ and fit the $\Delta E$ spectra simultaneously for different sub-decay processes. The obtained branching fraction for $B^{0} \rightarrow J / \psi \eta$ is $(12.2 \pm 1.7 \pm 0.9) \times 10^{-6}$ and is consistent with our previous measurement. However, the fits to $B^{0} \rightarrow J / \psi \eta^{\prime}$ do not have significant $B$ yields and the results are shown in Fig. 2. We set the corresponding upper limit, $\mathscr{B}\left(B^{0} \rightarrow J / \psi \eta^{\prime}\right)<1.1 . \times 10^{-5}$, at the $90 \%$ confidence level.
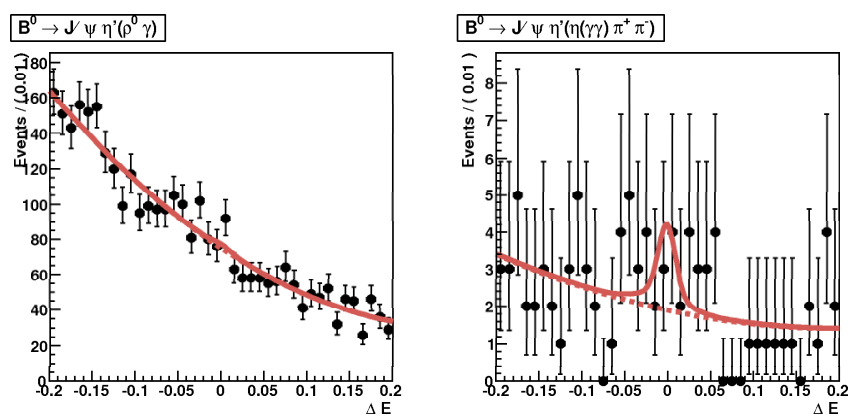

Figure 2: The $\Delta E$ distributions for $B^{0} \rightarrow J / \psi \eta^{\prime}$ with $\eta^{\prime} \rightarrow \rho^{0} \gamma$ and $\eta^{\prime} \rightarrow \eta \pi^{+} \pi^{-}$. The solid curve is the over all fit result and the dashed line indicates background.

\section{Acknowledgments}

The author wish to thank the KEKB accelerator group for the excellent operation of the KEKB accelerator. This work is supported by the National Science Council of the Republic of China under the grant NSC-99-2112-M-002-006-MY3.

\section{References}

[1] Throughout this report, inclusion of charge conjugate mode is always implied unless otherwise stated.

[2] P. Chen et al. (Belle Collaboration), Phys. Rev. D84, 071501 (2011).

[3] M.Z. Wang et al. (Belle Collaboration), Phys. Lett. B617, 141 (2005).

[4] M.Z. Wang et al. (Belle Collaboration), Phys. Rev. D76, 052004 (2007).

[5] C. Chen et al., Phys. Rev. D78, 054016 (2008).

[6] A. Abashian et al. (Belle Collaboration), Nucl. Instr. and Meth. A479, 117 (2002).

[7] M.C. Chang et al. (Belle Collaboration), Phys. Rev. Lett. 98 , 131803 (2007).

[8] F. Ambrosino et al. (KLOE Collaboration), J. High Energy Phys. 07 , 105 (2009).

[9] S. Kurokawa and E. Kikutani, Nucl. Instr. and Meth. A499, 1 (2003) and other papers included in this Volume.

[10] G.J. Feldman and R.D. Cousins, Phys. Rev. D 57, 3873 (1998). 